\title{
Multi-Threaded Evolution of the Data-Logging System of the ATLAS Experiment at CERN
}

\section{Tommaso Colombo ${ }^{a, b} \quad$ Wainer Vandelli ${ }^{b}$}

a Università degli Studi di Pavia

$$
{ }^{b} \text { CERN }
$$

IEEE Nuclear Science Symposium, 27 October 2011 


\section{The ATLAS Trigger \& DAQ System}

Peak Event rates

$20 \mathrm{MHz}$

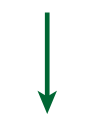

$75 \mathrm{kHz}$

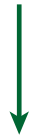

$\sim 5 \mathrm{kHz}$

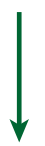

$\sim 600 \mathrm{~Hz}$

\section{Trigger}

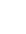

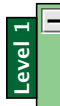

Custom

Hardware
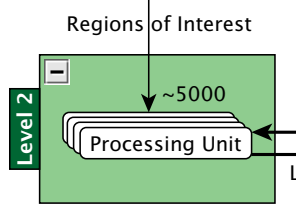

L2 Accept

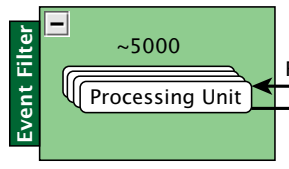

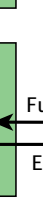

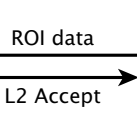
Network

\section{Level 1 Accept}
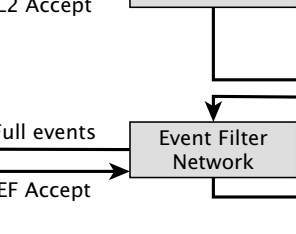

Event Filter Network

\section{Data Collection}
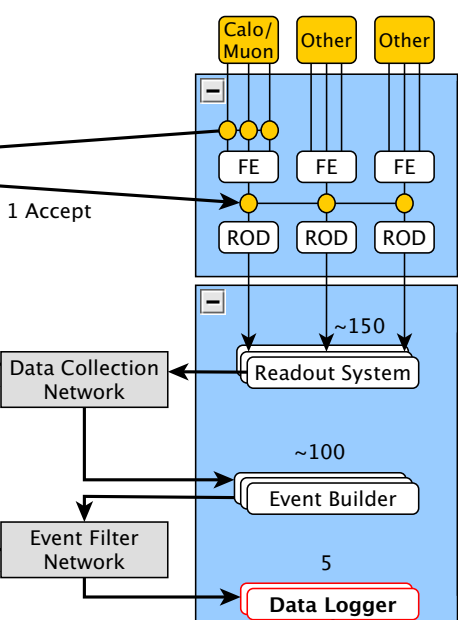

$\sim 100$

Event Builder

5

Data Logger

\section{CEERN}

Peak

Data rates

ATLAS

Event

$1.2 \mathrm{MB} / 50 \mathrm{~ns}$

( 24 TB/s)

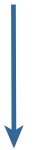

$\sim 90 \mathrm{~GB} / \mathrm{s}$<smiles>C=CCCC</smiles>

$\sim 6 \mathrm{~GB} / \mathrm{s}$

$\downarrow$

$\sim 700 \mathrm{MB} / \mathrm{s}$ 


\section{The current Data Logging system: overview}

\section{Purpose}

5 PCs receive data from the Event Filter system and write it to local disks. Each event is:

- analyzed to determine the "tags" applied by the EF trigger

- "processed"

- written to the appropriate file(s) according to the tags

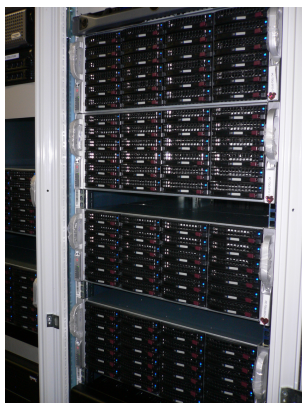

- The event tags are determined by the trigger algorithms at the EF

- Every event is written to multiple files, one per each of its tags

- File checksum is calculated while writing $\Rightarrow \mathrm{CPU}$-intensive! 


\section{The current Data Logging system: overview}

\section{Purpose}

5 PCs receive data from the Event Filter system and write it to local disks. Each event is:

- analyzed to determine the "tags" applied by the EF trigger

- "processed"

- written to the appropriate file(s) according to the tags

\section{Details}

- The event tags are determined by the trigger algorithms at the EF

- Every event is written to multiple files, one per each of its tags

- File checksum is calculated while writing $\Rightarrow \mathrm{CPU}$-intensive! 


\section{The current Data Logging system: limitations}

- The current Data Logger implementation is essentially single-threaded:

- multiple threads receive the events from the EF

- a single thread does the processing and writing

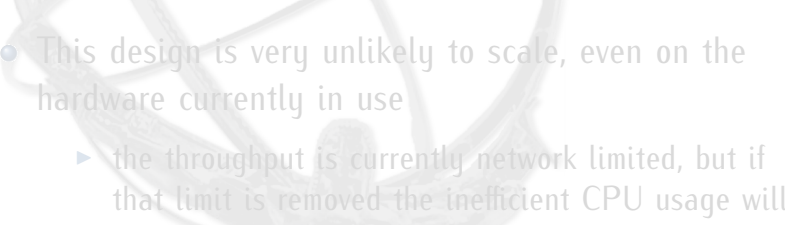

Network I/O Thread put event

Events Q.

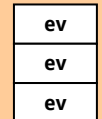

get event

Processing and

Writing Thread 


\section{The current Data Logging system: limitations}

- The current Data Logger implementation is essentially single-threaded:

- multiple threads receive the events from the EF

- a single thread does the processing and writing

- This design is very unlikely to scale, even on the hardware currently in use

- the throughput is currently network limited, but if that limit is removed the inefficient CPU usage will be the bottleneck

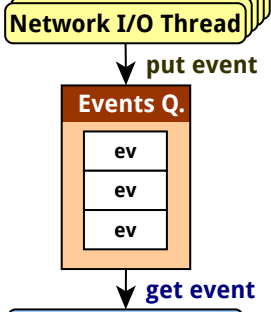

Processing and

Writing Thread 


\section{The current Data Logging system: limitations}

- The current Data Logger implementation is essentially single-threaded:

- multiple threads receive the events from the EF

- a single thread does the processing and writing

- This design is very unlikely to scale, even on the hardware currently in use

- the throughput is currently network limited, but if that limit is removed the inefficient CPU usage will be the bottleneck

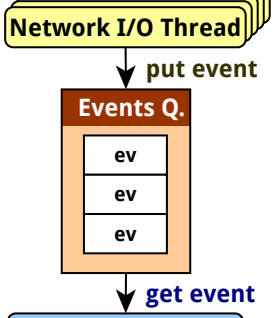

Processing and

Writing Thread

- It is a major blocker for the addition of new features requiring more CPU power than a single core can provide

- due to this event-level data compression currently needs to be performed off-line, as an additional step 


\section{New design: general considerations}

- The workload is embarrassingly parallel: the incoming data are already divided in events

The raw data file format is strictly sequential
$\Rightarrow I t$ is impossible to do concurrent writes to the same file

The sequential file format is necessary to calculate the overall file checksum before

writing to disk, aiding in the detection of write errors 


\section{New design: general considerations}

- The workload is embarrassingly parallel: the incoming data are already divided in events

\section{Constraint}

The raw data file format is strictly sequential

$\Rightarrow$ It is impossible to do concurrent writes to the same file

The sequential file format is necessary to calculate the overall file checksum before writing to disk, aiding in the detection of write errors 


\section{New design: general considerations}

- The workload is embarrassingly parallel: the incoming data are already divided in events

\section{Constraint}

The raw data file format is strictly sequential

$\Rightarrow$ It is impossible to do concurrent writes to the same file

The sequential file format is necessary to calculate the overall file checksum before writing to disk, aiding in the detection of write errors

- Multiple events can be written to different raw data files concurrently, but no more than one event can be written to each data file at once 


\section{New design: idea!}

- Split the workload in tasks

- For each event:

- one task does the processing

- multiple tasks do the writing

(for each tag, a task writes the event to the corresponding file). 


\section{New design: idea!}

- Split the workload in tasks

- For each event:

- one task does the processing

- multiple tasks do the writing

(for each tag, a task writes the event to the corresponding file).

- Use a single thread pool to execute the tasks

- Schedule the tasks cleverly to avoid locking 


\section{New design: idea!}

- Split the workload in tasks

- For each event:

- one task does the processing

- multiple tasks do the writing (for each tag, a task writes the event to the corresponding file).

- Use a single thread pool to execute the tasks

- Schedule the tasks cleverly to avoid locking

- At any given time:

- any number of processing tasks can run

- for each raw data file, only one task writing to it can run 


\section{New design: finally, a diagram!}

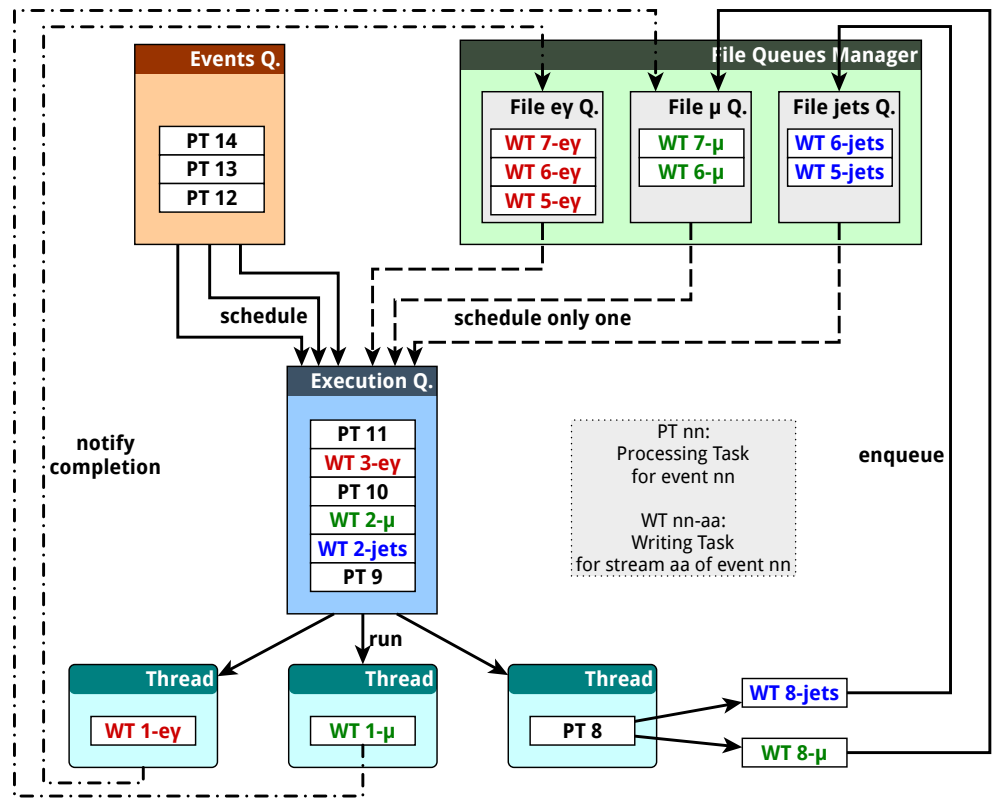




\section{New design: Intel Threading Building Blocks}

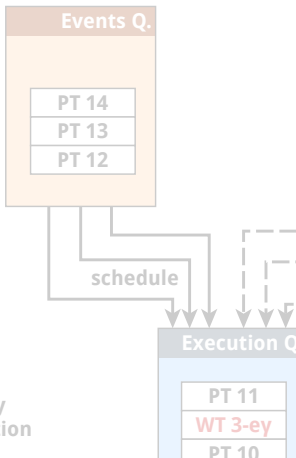

The new design was implemented using (and inspired by) the open source $\mathrm{C}++$ library Intel Threading Building Blocks 


\section{New design: Intel Threading Building Blocks}

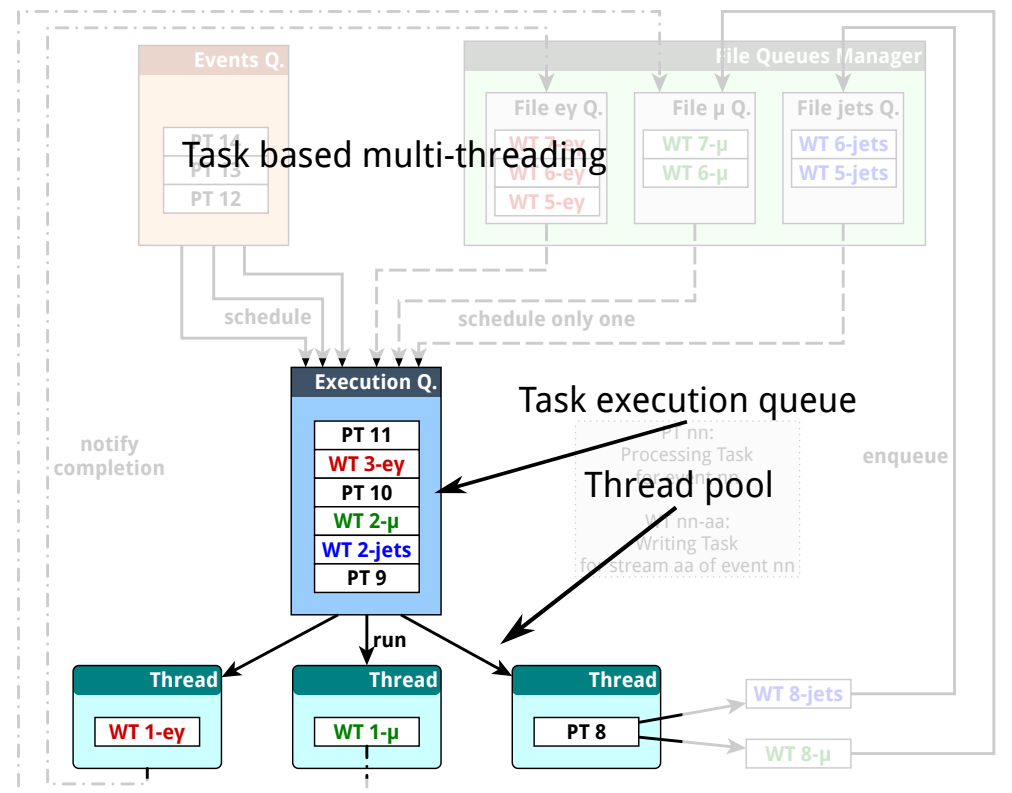




\section{New design: Intel Threading Building Blocks}

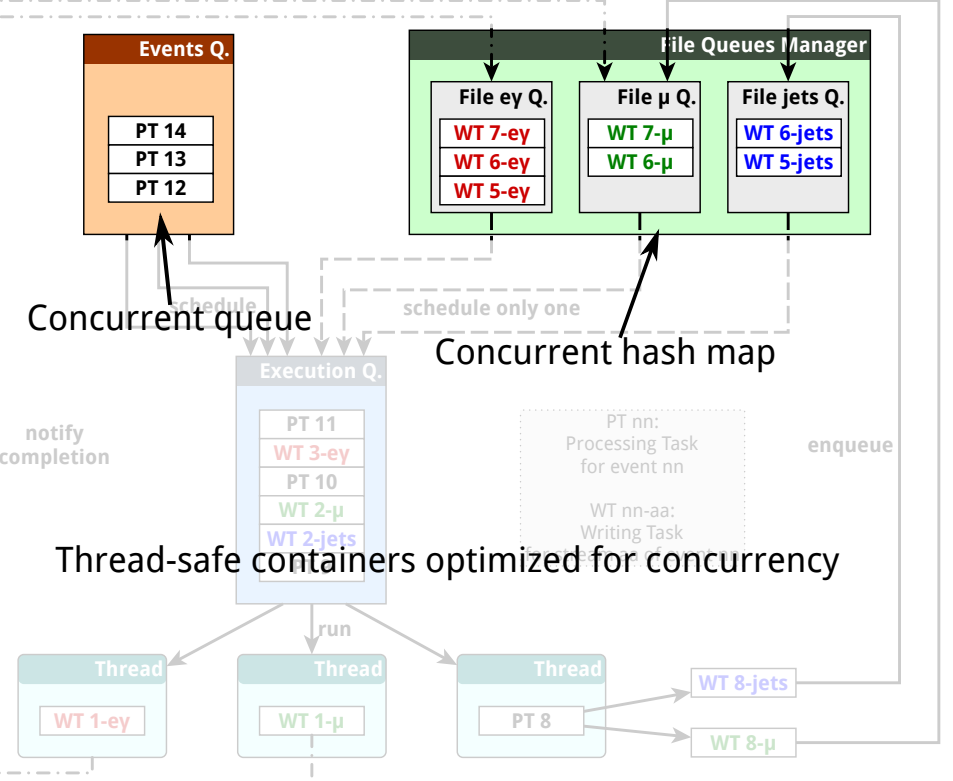




\section{Testing: performance \& resource utilization}

- The new implementation was tested and compared with the old one in a testbed sporting older hardware with respect to the production system

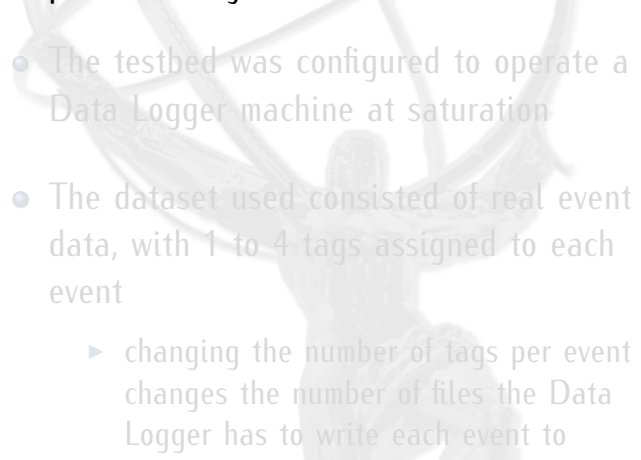

\section{Testbed Data Logger PC}

\begin{tabular}{|c|}
\hline 2x dual core Xeon 5130 \\
\hline 4 GB \\
\hline 3x 3ware RAID5 array \\
\hline 2x GbE NIC \\
\hline
\end{tabular}

\section{Production Data Logger PC}

\begin{tabular}{|c|}
\hline 2x quad core Xeon E5520 \\
\hline 24 GB \\
\hline 3x Adaptec RAID5 array \\
\hline 2x GbE NIC \\
\hline
\end{tabular}




\section{Testing: performance \& resource utilization}

- The new implementation was tested and compared with the old one in a testbed sporting older hardware with respect to the production system

- The testbed was configured to operate a Data Logger machine at saturation

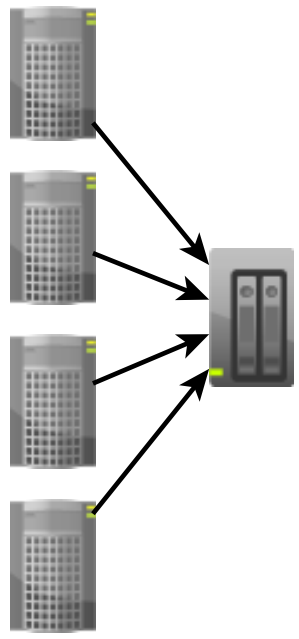




\section{Testing: performance \& resource utilization}

- The new implementation was tested and compared with the old one in a testbed sporting older hardware with respect to the production system

- The testbed was configured to operate a Data Logger machine at saturation

- The dataset used consisted of real event

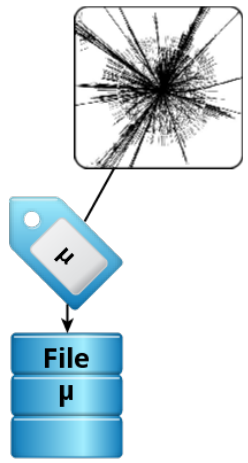
data, with 1 to 4 tags assigned to each event

- changing the number of tags per event changes the number of files the Data Logger has to write each event to 


\section{Testing: performance \& resource utilization}

- The new implementation was tested and compared with the old one in a testbed sporting older hardware with respect to the production system

- The testbed was configured to operate a Data Logger machine at saturation

- The dataset used consisted of real event data, with 1 to 4 tags assigned to each

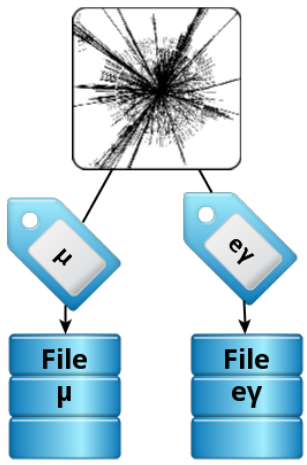
event

- changing the number of tags per event changes the number of files the Data Logger has to write each event to 


\section{Testing: performance \& resource utilization}

- The new implementation was tested and compared with the old one in a testbed sporting older hardware with respect to the production system

- The testbed was configured to operate a Data Logger machine at saturation

- The dataset used consisted of real event data, with 1 to 4 tags assigned to each event

- changing the number of tags per event changes the number of files the Data Logger has to write each event to

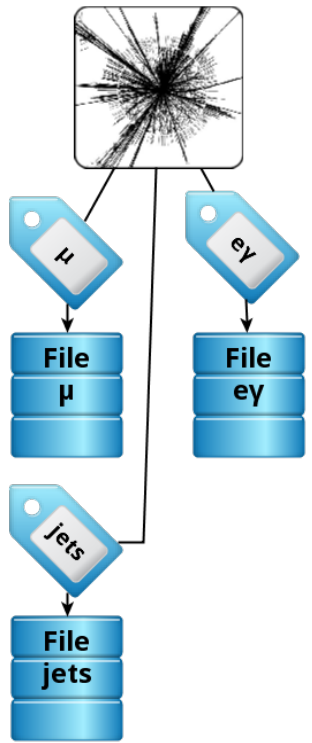




\section{Testing: performance \& resource utilization}

- The new implementation was tested and compared with the old one in a testbed sporting older hardware with respect to the production system

- The testbed was configured to operate a Data Logger machine at saturation

- The dataset used consisted of real event data, with 1 to 4 tags assigned to each event

- changing the number of tags per event changes the number of files the Data Logger has to write each event to

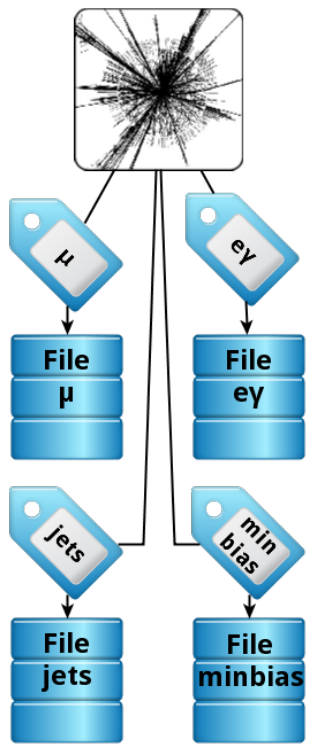




\section{Testing: performance \& resource utilization}

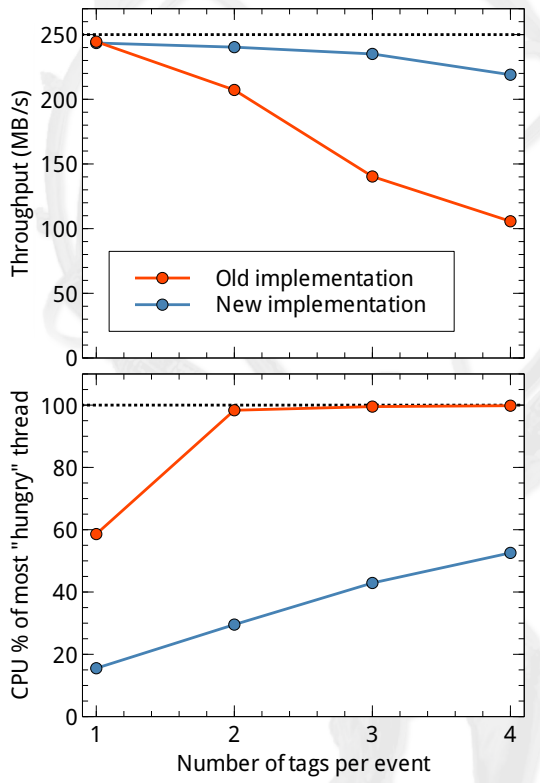

The "hard" limit on the throughput of a single Data Logger is given by the network bandwidth: $2 \mathrm{~Gb} / \mathrm{s} \simeq 250 \mathrm{MB} / \mathrm{s}$

Old single-threaded implementation

- Can operate at network saturation only for a single tag per event

- Above 2 tags per event, the load generated by its single thread exceeds what a single CPU core can take

- The throughput decreases accordingly 


\section{Testing: performance \& resource utilization}

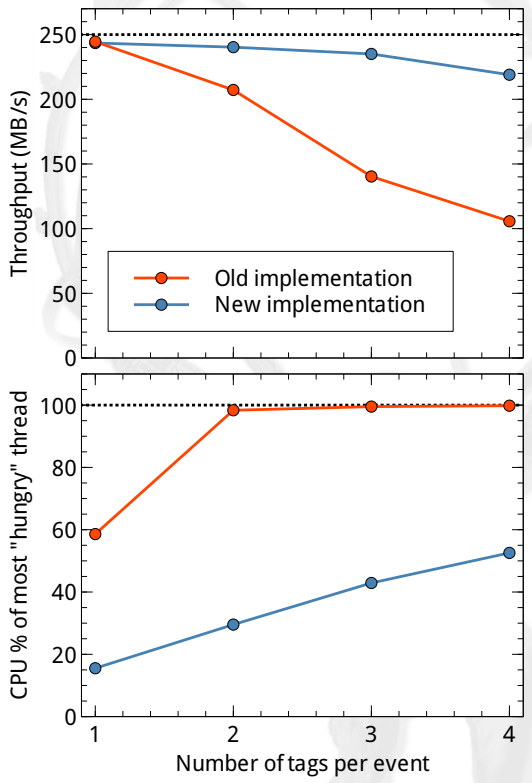

The "hard" limit on the throughput of a single Data Logger is given by the network bandwidth: $2 \mathrm{~Gb} / \mathrm{s} \simeq 250 \mathrm{MB} / \mathrm{s}$

New multi-threaded implementation

- The throughput is almost unaffected by the load

- Its 4 threads spread the workload on the 4 CPU cores: any of them does not use more than $60 \%$ of a core

- Leaves plenty of legroom for additional CPU intensive processing $\Rightarrow$ compression 


\section{Testing: scalability}

- On-line event compression radically changes the landscape:

- throughput is much lower

- all workloads saturate the CPU

- compr. time $\gg$ checksum time

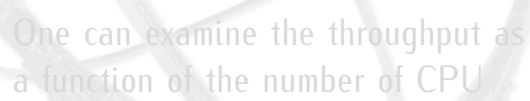

cores (threads) used

scaling is (almost) linear

- The throughput of the compression alone was measured on the testbed and on the production machines

- the results suggest that enabling on-line event compression on the production machine is feasible

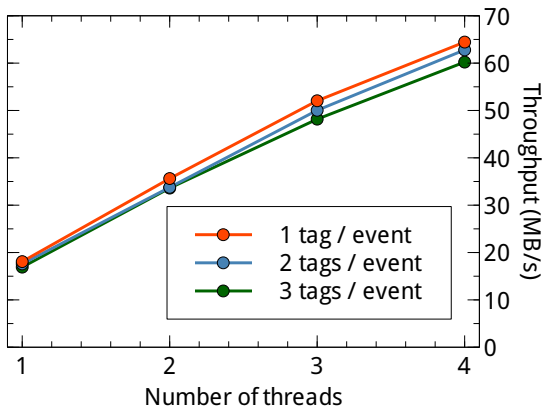

\begin{tabular}{|c|c|}
\hline $\begin{array}{c}\text { Data Logger } \\
\text { machine }\end{array}$ & $\begin{array}{r}\text { Compression-only } \\
\text { throughput }\end{array}$ \\
\hline \begin{tabular}{|c|} 
Testbed \\
4 threads
\end{tabular} & $71 \mathrm{MB} / \mathrm{s}$ \\
\hline $\begin{array}{c}\text { Production } \\
4 \text { threads }\end{array}$ & \\
\hline Production & $161 \mathrm{MB} / \mathrm{s}$ \\
8 threads & \\
\hline
\end{tabular}




\section{Testing: scalability}

- On-line event compression radically changes the landscape:

- throughput is much lower

- all workloads saturate the CPU

- compr. time $\gg$ checksum time

- One can examine the throughput as a function of the number of CPU cores (threads) used

- scaling is (almost) linear

- The throughput of the compression alone was measured on the testbed

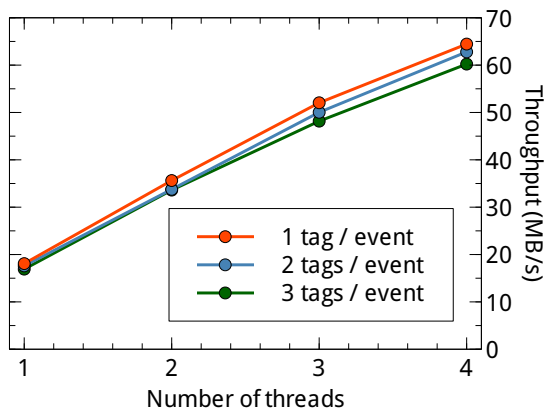
and on the production machines

> the results suggest that enabling on-line event compression on the

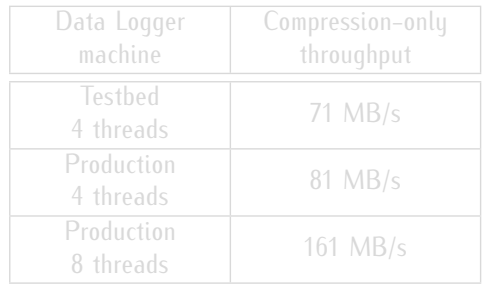

production machine is feasible 


\section{Testing: scalability}

- On-line event compression radically changes the landscape:

- throughput is much lower

- all workloads saturate the CPU

- compr. time $\gg$ checksum time

- One can examine the throughput as a function of the number of CPU cores (threads) used

- scaling is (almost) linear

- The throughput of the compression alone was measured on the testbed and on the production machines

- the results suggest that enabling on-line event compression on the production machine is feasible

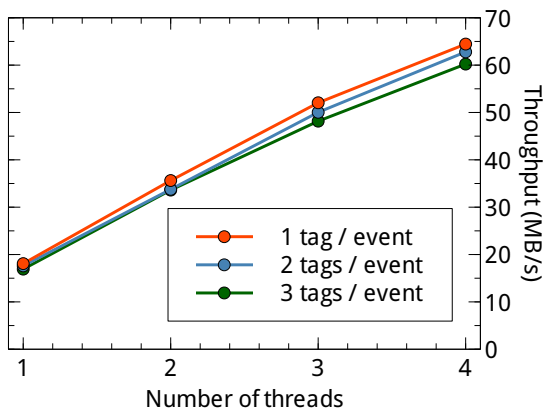

\begin{tabular}{|c|c|}
\hline $\begin{array}{c}\text { Data Logger } \\
\text { machine }\end{array}$ & $\begin{array}{c}\text { Compression-only } \\
\text { throughput }\end{array}$ \\
\hline \hline $\begin{array}{c}\text { Testbed } \\
4 \text { threads }\end{array}$ & $71 \mathrm{MB} / \mathrm{s}$ \\
\hline $\begin{array}{c}\text { Production } \\
4 \text { threads }\end{array}$ & $81 \mathrm{MB} / \mathrm{s}$ \\
\hline $\begin{array}{c}\text { Production } \\
8 \text { threads }\end{array}$ & $161 \mathrm{MB} / \mathrm{s}$ \\
\hline
\end{tabular}




\section{Conclusions}

- A novel design for the ATLAS Data Logging application was implemented and thoroughly tested

crest results are promising: it was demonstrated that it exploits the full power of moden CPUS 


\section{Conclusions}

- A novel design for the ATLAS Data Logging application was implemented and thoroughly tested

- Test results are promising: it was demonstrated that it exploits the full power of modern CPUs 


\section{Conclusions}

- A novel design for the ATLAS Data Logging application was implemented and thoroughly tested

- Test results are promising: it was demonstrated that it exploits the full power of modern CPUs

- The design will be fully validated using the full DAQ system with a view to adopting it for the 2012 data taking period

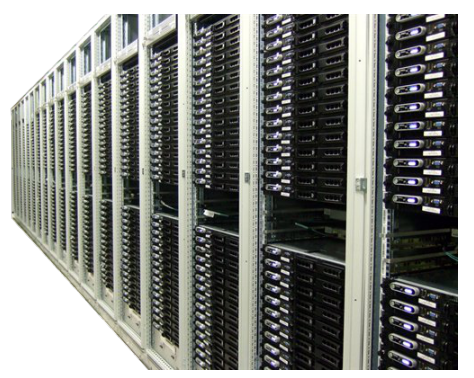




\section{Backup}




\section{Other design constraints}

Operations are driven by the received event data

The Data Logger can only rely on the information it gathers by examining the received events

- Cannot assume that the rate of received events is somehow balanced across the spectrum of the possible tags

- The flow of events with one tag can vary during a run and even stop completely 


\section{Other design constraints}

Operations are driven by the received event data

The Data Logger can only rely on the information it gathers by examining the received events

No assumptions about the data flow

- Cannot assume that the rate of received events is somehow balanced across the spectrum of the possible tags

- The flow of events with one tag can vary during a run and even stop completely 


\section{Other possible designs: thread pool with locking}

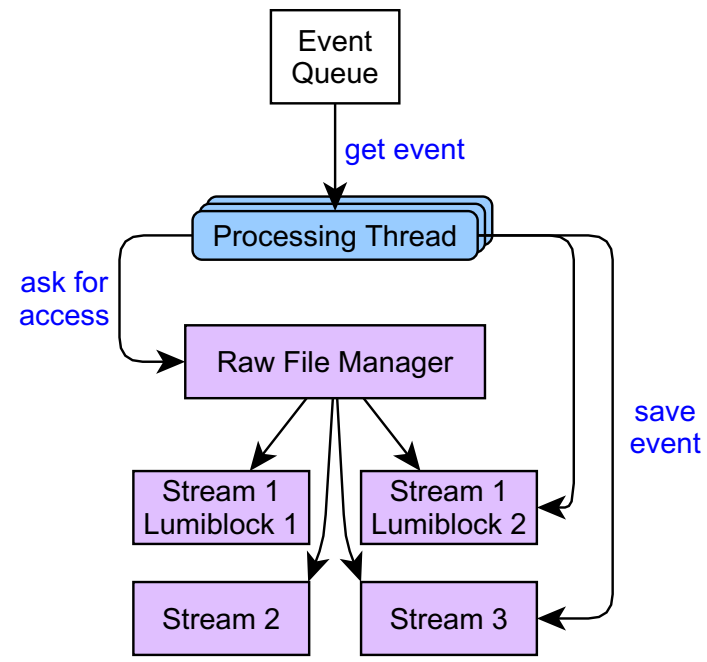




\section{Other possible designs: chain of responsibility}

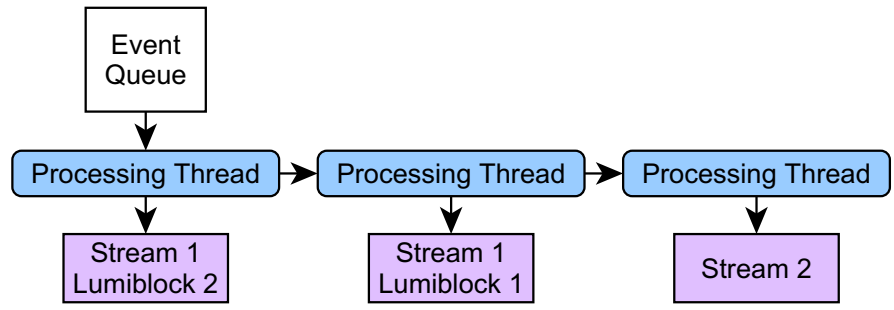

Risk of starvation! 


\section{Other possible designs: one thread pool per file}

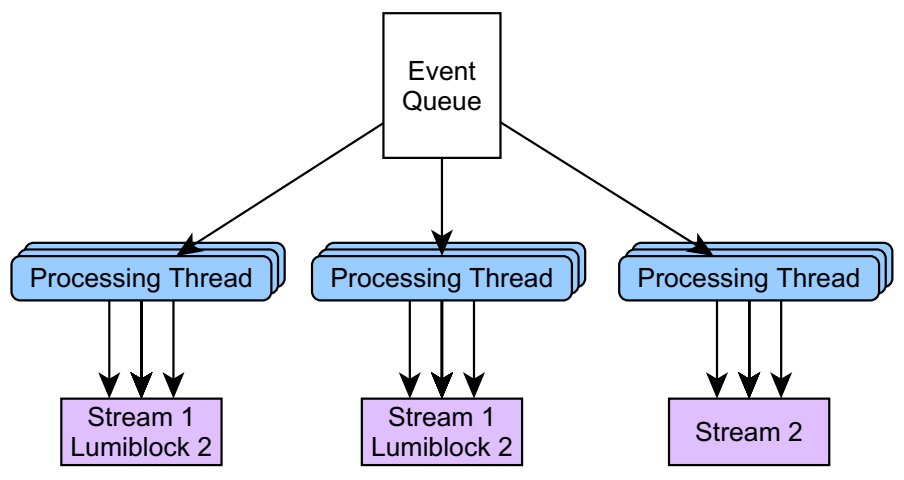

Too many threads! 